\title{
Recent progress of optical functional nanomaterials based on organoboron complexes with $\beta$-diketonate, ketoiminate and diiminate
}

\author{
Kazuo Tanaka and Yoshiki Chujo
}

The synthesis and application of organoboron complexes are a highly relevant topic owng to their unique characteristics. Based on their emissive properties, these complexes have been used to make novel optical materials and devices; boron $\beta$-diketonate is a simple and robust organoboron complex. From a series of recent studies, unique and versatile optical properties have been reported. In this review, we introduce the results of primarily recent studies on boron diketonate and related compounds containing polymers and particularly explain their optical properties. Initially, the multi-emission of boron diketonate derivatives and its application to biotechnology are explained. Next, the formation of nanostructures and its emission properties are demonstrated. The modulation of optical properties by mechanical stress is also presented. Finally, recent progress in the development of solid-emissive materials are shown with boron diketonates and their derivatives, which have aggregation-induced emission properties. The versatility of boron diketonates as a building block for the preparation of functional optical materials is the focus of this review.

NPG Asia Materials (2015) 7, e223; doi:10.1038/am.2015.118; published online 6 November 2015

\section{INTRODUCTION}

Using heteroatoms or organometallic complexes for material designs, unusual functions due to the effects of each element can be expected. From the synergetic effects between a heteroatom and a conjugation system, significant characteristics that are not attainable using only $\mathrm{C}$, $\mathrm{H}, \mathrm{O}$, and $\mathrm{N}$ are often obtained. Thus we can say that heteroatoms or organometallic complexes are attractive building blocks for creating high-performance materials based on molecular designs. We have recently proposed the new 'element-block' concept, which describes the various groups of heteroatoms. ${ }^{1}$ With this concept, organoboronconjugated molecules can act as a versatile platform for expressing unique optical properties as a representative example of element blocks. Boron belongs to the thirteenth group on the periodic table and has one less electron than carbon. By connecting a conjugation system with the tri-coordinated boron atom, the conjugation can be elongated by the boron atom owing to the vacant $\mathrm{p}$ orbital; this can occur even in the polymers. ${ }^{2}$ Various intriguing characteristics have been obtained, such as low-lying LUMO orbitals, an improvement of electron-accepting ability and a bathochromic shift of the absorption and emission spectra. These low-lying LUMO levels are advantageous for the design of organic light-emitting devices. ${ }^{3}$ Because of the strong electron affinity of tri-coordinated boron, conjugation systems can show strong affinities toward Lewis bases. Based on this fact, chemical sensors have been constructed for anion sensing. ${ }^{2}$ Even rare electronic states such as anti-aromaticity have been achieved with the borafluorene structure with the tri-coordinate state of boron. ${ }^{4}$ However, because of the intrinsic instability of the tri-coordinate state of boron, synthesis and material usages are still challenging. Compared with tri-coordinate boron, the organoboron complexes with tetracoordinate boron generally exhibit higher stabilities. To date, various types of luminescent materials involving tetra-coordinate boron have been reported. For example, boron dipyrromethene derivatives are well known to have superior optical properties, such as high lightabsorption and -emission abilities, sharp spectra and high photostabilities and are used for a wide variety of applications in both material science and biotechnology. ${ }^{5}$ Photoisomerization properties have also been observed with organoboron compounds. ${ }^{6}$

Boron diketonates (Scheme 1) with tetra-coordinate boron atom represent a classic organoboron complex. Because of the wide acceptability of the $\beta$-diketonate ligand, various metal complexes including boron can be readily constructed. ${ }^{7}$ Boron diketonates exhibit high stabilities toward oxygen and moisture; only under strong alkaline conditions or in the presence of an excess amount of fluoride anion does degradation of boron diketonates occur. Most boron diketonates exhibit difluoride forms and are highly planar. Boron diketonates have been used as building blocks for constructing nanoarchitectures. After complexation, certain optical properties can be obtained using boron diketonates. For example, 1,3-diphenyl-2,3propanedione, which is the ligand moiety of boron diketonate that is the primary focus in this review, exhibits marginal emission in the 


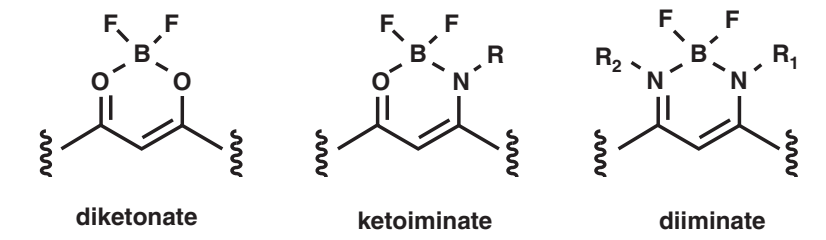

Scheme 1 Chemical structures of boron diketonate, ketoiminate and diiminate.

visible region. After the introduction of difluoroboron, a bright emission can be observed from the resulting boron diketonate complex (that is, boron difluoride dibenzoylmethane) in the blue region. Because of the substituent effect on both sides of the aryl groups, the optical properties of the material can be tuned. Since the earlier reports on the optical properties, versatile functions have been reported. ${ }^{8}$ Boron diketonates commonly have weak electron-accepting abilities; therefore, Lewis bases often interact with boron diketonate complexes. By connecting with electron-donating units, the intermolecular charge-transfer state should be formed, resulting in strong emissive properties. By conjugation with other functional units and by introducing the material into the conjugation systems, novel luminescence properties have been reported and will be described later in this review.

The emission properties of boron diketonates in the solution state have been comprehensively examined since the early stage of research in this field of study. ${ }^{8}$ The emission behaviors of boron diketonates can be sufficiently explained by conventional theory; however, more recently, unusual optical properties have been discovered. Particularly in the condensed state or using intermolecular interactions, a series of unique characteristics have been observed. In this review, these luminescent properties of boron diketonates and related compounds such as ketoiminates and diiminates are investigated in detail. Recent topics from the preparation of optical materials to practical application are presented. Initially, the multi-emission of boron diketonate derivatives is introduced. Typically, a single emission band is obtained from the molecule. Conversely, some boron diketonates can provide dual emission bands in a given environment. This review describes these results and the implications regarding applications they can have in biotechnology. Next, the formation of nanostructures is demonstrated. Precise controls for the formation of self-assembly structures were accomplished. We also mention the characteristics of these nanostructures. Conventional organic emissive dyes can emit only in diluted solutions. It was found that some boron diketonate and nitrogen-substituted complexes can exhibit significant emissions even in the solid state. Additionally, the differences in the optical properties between the crystal and amorphous states were obtained. Based on this alteration, the modulation of optical properties by mechanical stress was also achieved. Finally, recent progress of the development of solidemission materials is shown using boron diketonates and their derivatives with aggregation-induced emission (AIE) properties. The versatility of boron diketonates is described in this review.

\section{LUMINOGENS FOR DUAL EMISSIONS}

Dual-emission materials are feasible owing to their significant potential for use in organic opto-electronic devices, such as displays and chemosensors. By maintaining or creating multiple excited states in the material, the multiple emission bands in the different wavelength regions can be achieved independently. For example, by loading red, green and blue chromophores into polymeric matrices, white-lightemissive materials that are used for illumination and a back light in

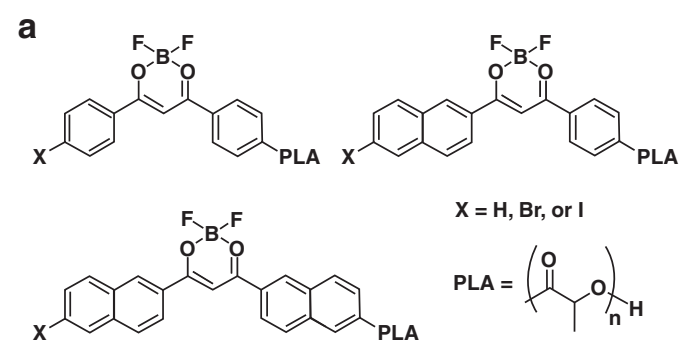

b

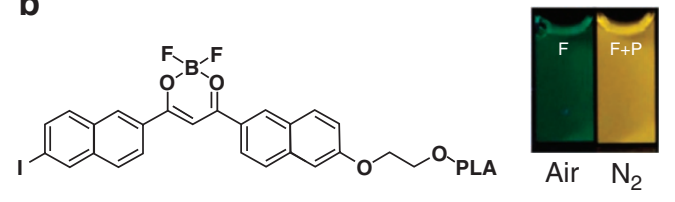

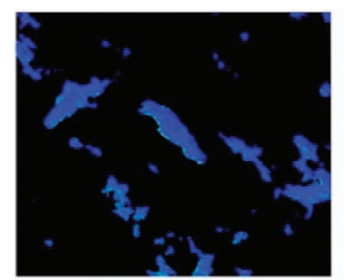

Hypoxia

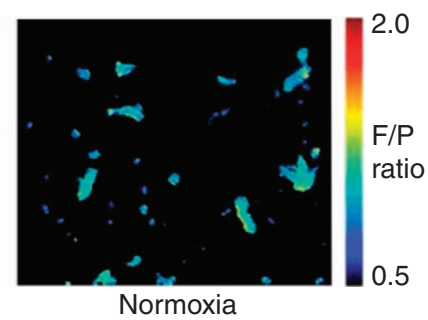

Figure 1 (a) Chemical structures of dual-emissive boron diketonates. (b) Picture of the solutions under aerobic and anaerobic conditions and oxygen-level mapping with the cell. Panel (b) was adapted with permission from Zhang et al. ${ }^{11}$ Copyright 2015 American Chemical Society.

the liquid crystal-based display can be readily obtained..$^{9}$ By modulating the mixing ratio of each luminescent component, precise color tuning is possible. In these mixture systems, the loss in the intensity of relatively higher energy colors (for example, blue or green) due to the energy transfer can be canceled by modulating the mixing ratio. An optical sensor based on a fluorescent, artificial nucleobase has also been created for monitoring structural alterations in DNA. ${ }^{10}$ The emission band for intramolecular charge transfer from the modified, pyrene-substituted, fluorescent nucleobase was observed in the solution. Conversely, when the modified nucleobase is located in the duplex, the modified pyrene moiety should be intercalated with stacking. Subsequently, suppression of the molecular motion at the excited state should occur. Finally, the dual-emission property from the intrinsic intramolecular charge transfer and locally excited states can be detected, resulting in a color change. This phenomenon is applicable for the simple detection of DNA hybridization. As explained above, multi-emission materials can be fabricated using the mixture system. However, these materials would present the desired property only under certain conditions, because there are potential difficulties in material preparation, such as suppressing the energy transfer, concentration quenching and phase separation of the components. Thus dual-emission materials, particularly those based on single dye molecules, should be useful for applications in various fields.

Fraser and colleagues have recently reported a series of the modified boron diketonates with polymers and their dual-emissive properties (Figure 1). It was shown that these materials are applicable for oxygenlevel mapping with cellular tissues and for in vivo imaging studies of tumor hypoxia. ${ }^{11,12}$ Difluoroboron-substituted diketonates can show the strong fluorescence that is attributable to $\pi-\pi^{*}$ transition or intramolecular charge transfer. Boron diketonates intrinsically exhibit phosphorescence. By introducing a halogen atom on one side of the 


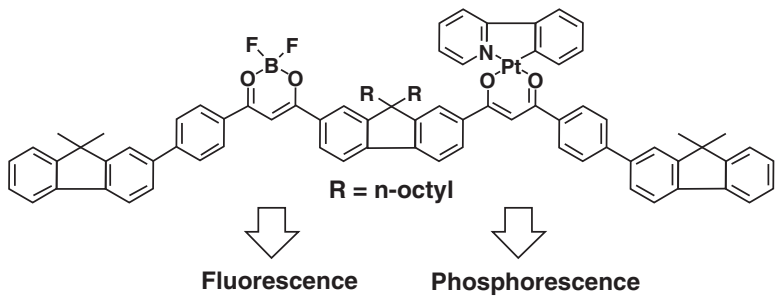

Scheme 2 Chemical structure and emission mechanism of the dyad with boron and platina diketonates.

molecule, the phosphorescence property can be significantly enhanced via the internal heavy-atom effect. Finally, these modified diketonates show the dual-emission property via fluorescence and phosphorescence. ${ }^{13}$ Under aerobic conditions, only phosphorescence is quenched. By calculating the intensity ratio between the fluorescence and phosphorescence, and fitting with the standard line, the local oxygen level around the diketonate can be estimated. For example, difluoroboron dinaphthoylmethane polylactides were synthesized, and halogens (for example, bromine and iodine) were substituted. ${ }^{14}$ All materials showed fluorescence with a peak at approximately $510 \mathrm{~nm}$ (that is, green). In addition, the iodine-substituted diketonate clearly showed phosphorescence with a peak at approximately $560 \mathrm{~nm}$ (that is, yellow). The nanoparticles were prepared with these materials, and the oxygen levels were visually discriminated using ratiometric probes.

The dual-emission property was obtained by the combination of boron diketonate and organometallic diketonate with different types of cations. The diketonate-based dyad composed of boron and platinum complexes was synthesized (Scheme 2). ${ }^{15}$ Dual emissions were observed from the synthesized dyes by individually obtaining the emissions from each organometallic complex. In acetonitrile, the dyad presented two peaks at 541 and $579 \mathrm{~nm}$, which can be attributable to the fluorescence of boron and the phosphorescence of platinum moieties, respectively. Based on lifetime measurements, it was confirmed that the emission of the dyad primarily involves two elements, both with short life times, which can be attributable to fluorescence, and long life times, which can be attributed to the phosphorescence. Although the fluorescence quantum yields of boron diketonates were reduced owing to the introduction of platinum via the heavy-metal effect in this study, the mixing spectra with the emissions generated from the different spin multiplicities were obtained. Organometallic diketonate complexes can show unique and varied types of optical and electric properties. This strategy could be applicable when developing new types of optical materials using additional combinations of different pairs of metal elements.

\section{BUILDING BLOCKS FOR CONSTRUCTING NANOSTRUCTURES AND SUPRAMOLECULES}

Optical properties are closely related to the electronic structure of the molecules in a compound; thus chemical modification is the typical method used to modulate these properties. Conversely, in the condensed or solid states, molecular distribution and morphology should significantly influence the electronic structures via intermolecular interactions. From this perspective, there is a possibility to create new optical properties using the nanostructures and supramolecules composed of boron diketonates, leading to the development of new functional materials. In this section, the formation of nanostructures and supramolecules and their optical properties are shown.

Boron diketonates can work as a mesogen to form liquid crystals with emission properties. Therefore, boron diketonates can be expected to work not only as building blocks for constructing nanomaterials but also as luminogens in these nanostructures. Turanova et al. ${ }^{16,17}$ reported liquid-crystal polymorphism with 2-alkyl-substituted boron diketonates with their luminescence properties. From the investigation of the influence of the ligand structure on the liquid-crystal properties, it was found that the dodecyloxybenzoate-substituted complex exhibited a mesophase. The typical emission band between 450 and $650 \mathrm{~nm}$ from the boron diketonate moiety was observed. Cano and colleagues obtained the series of luminescent liquid crystals based on boron diketonates. ${ }^{18,19}$ Asymmetric complexes were prepared with 1,3-alkoxyphenyl-substituted diketonate ligands to create boron difluoride. ${ }^{18}$ The emission bands with peaks at approximately $490 \mathrm{~nm}$ were observed in the solid state. In addition, mesophases were observed during the melting processes in the differential scanning calorimeter profiles. The existence of the banana-shaped liquid-crystal mesophases was present. This unique mesophase structure could have originated from the intrinsic shape of boron diketonate. Recently, the diketonate derivatives formed discotic liquid crystals that exhibit hexagonal, columnar mesophases from the polycatenar 1,3-alkyloxyphenyl boron diketonate ${ }^{19}$ it was found that the mesophases were obtained at room temperature when the complexes possessed a highly symmetric structure. These molecules also showed high fluorescent emissions in the green region in the solid state. These bifunctional luminescent mesogens are said to be potential materials for the preparation of soft emission materials.

Molecular recognition using boron diketonate derivatives has been accomplished and used as a driving force to form nanostructures. Maeda et al. ${ }^{20}$ synthesized a series of 1,3-dipyrrole-substituted boron diketonates and reported on their molecular recognition behaviors with anion species (Figure 2). Dipyrrolyldiketone boron complexes, which bind anions via the inversion of two pyrrole rings, were synthesized. For example, in the presence of gallic acid derivatives, the formations of mesophases were observed. ${ }^{20}$ Highly ordered structures, including lamellar structures, were clarified from the structural analyses. It was demonstrated that these structural parameters could be modulated by the lengths of the alkyl chains in the anion species. In particular, from the fused complex with the bowl-shaped $\pi$-conjugated corannulene units, the emission property was observed from the assembly state. $^{21}$ Additionally, it was revealed that the electrical conductivity through the supramolecular structures could also be tuned. Similarly, based on the core $\pi$-planes in the presence of aliphatic side chains, supramolecular gels and thermotropic liquid crystals were obtained. ${ }^{22,23}$

Via conjugation with the commodity unit, additional functions could be incorporated. Various types of asymmetric complexes and tunable emissions have been reported. ${ }^{24-34}$ Emission colors can be modulated both in the solution and in solid states. In addition, a photo-responsive ketoiminate was obtained using a dithienylethene unit. ${ }^{35,36}$ The electronic structures could be altered by photo-irradiation, leading to the expression of photochromic behaviors (Scheme 3). By introducing the functional groups directly onto the boron atom in the diketonate complexes, these characteristics could be modulated. The dipyrrolyldiketone derivatives were modified with 1,1'-2-naphthol (BINOL) at the boron atom. ${ }^{37}$ Because of the chirality of the BINOL moiety, the complexes showed circular dichroism. In particular, by adding a chloride anion to the complex solutions, complexation occurred. Finally, it was observed that both the circular dichroism and circularly polarized luminescence (CPL) properties were enhanced; this is the first example of a chemical-stimuli-responsive CPL-active material. 

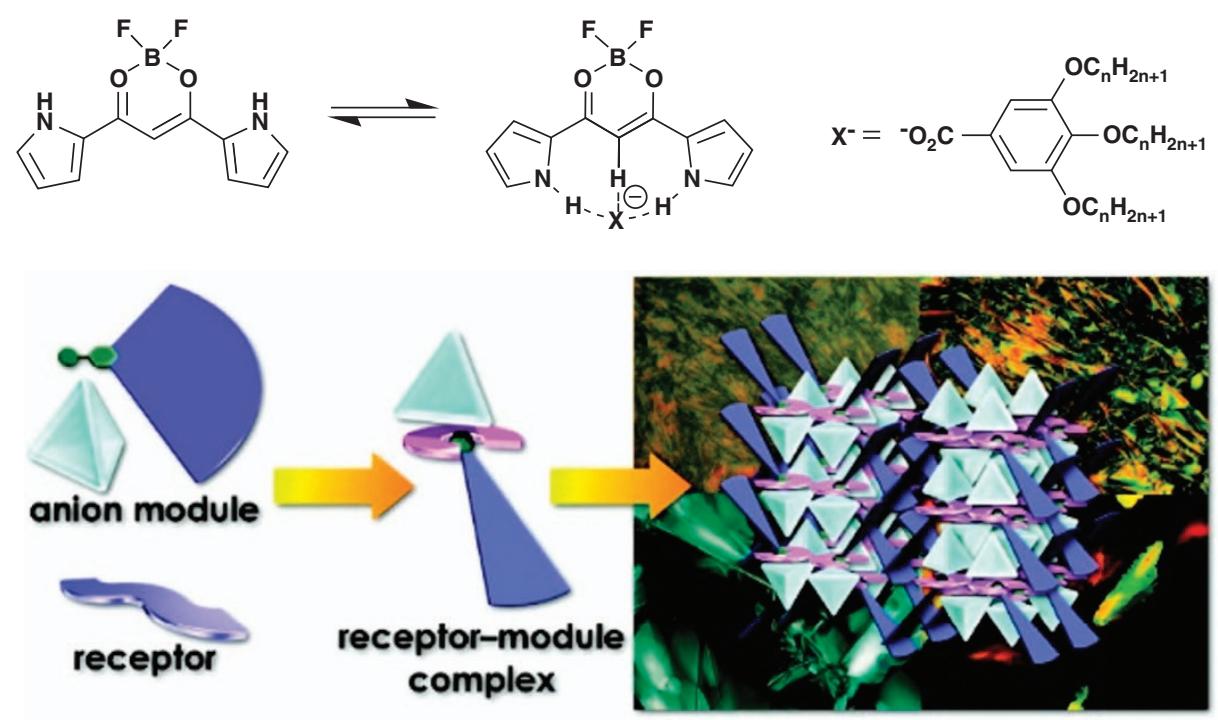

Figure 2 Schematic illustration for the formation of supramolecular structures based on anion recognition of $\pi$-conjugated boron diketonate receptor. Adapted with permission from Turanova et al. ${ }^{17}$ Copyright 2011 American Chemical Society.

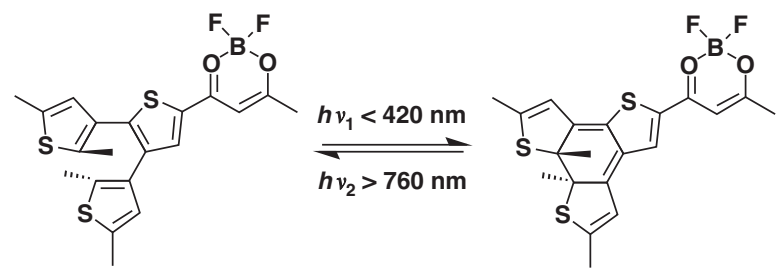

Scheme 3 Control of optical properties of the modified boron ketoiminate by light irradiation.

The tri-coordinate boron works as a strong Lewis acid owing to the electron affinity originated from the vacant $\mathrm{p}$ orbital. Therefore, the tri-coordinate, boron-containing conjugated molecules could be used as building blocks in anion sensors. Kumar and Thilagar ${ }^{38}$ synthesized the conjugated molecule with boron diketonate and triarylboron and monitored the changes in the optical properties of the material by adding anions. In the presence of fluoride and cyanide anions, ratiometric changes in the emission spectra were observed; particularly, the turn-on-type chemical sensor using a cyanide ion was shown to have a high selectivity. Typically, it was difficult to discriminate fluoride and other anion species. These data could be valid for the design of chemical sensors using other anion species.

\section{MECHANOFLUOROCHROMIC (MFC) MATERIALS}

MFC compounds that show switchable photoluminescent properties in response to mechanical stress are versatile materials for use in touch panels and physical stress sensors; several diketonates and ketoiminates have shown MFC behaviors. ${ }^{39-42}$ By grinding powder samples in the crystal state, materials typically convert into the amorphous state; the resulting molecular distribution would thus be randomized, and the degree of intermolecular interactions should be significantly different. As a result, changes in emission color or intensity can thus be observed. In the case of boron diketonate, hydrogen bonding was disrupted by grinding, resulting in a less ordered polymorph. ${ }^{43}$ As a result, MFC behaviors were observed. As mentioned above, the electronic structures of boron diketonates can be readily perturbed by the intermolecular interaction, leading to the alteration of the optical properties in the solid state. In this section, boron diketonates with MFC properties are introduced.

Difluoroboran avobenzone was synthesized, and their optical properties in the solid state were examined (Figure 3). ${ }^{44}$ Accordingly, narrow-band and morphology-dependent fluorescence was observed. In particular, it was found that mechanochromic fluorescence was reversibly recovered even at room temperature. The difluoroboron diketonate complex showed an emission band with a peak at $460 \mathrm{~nm}$. This blue-emission sample was ground; then the yellow color $\left(\lambda_{\mathrm{em}}=542 \mathrm{~nm}\right)$ was induced. After a few minutes, the yellow fluorescence faded, resulting in a green-blue emission. With heating, this process could be accelerated by several seconds; this implied that the molecular distribution and the morphology in the sample could be changed via the transition from the crystal to the amorphous state. As a result, different luminescence colors were observed.

More recently, the MFC system with diverse responses was reported (Figure 4). ${ }^{45}$ The luminescent organic crystal was prepared with boron diketonate. Block orange crystals were obtained from a dichloromethane solution of boron diketonate. By smashing the orange crystals with a stainless steel spoon, the fluorescence intensity increased. In addition, the spectrum shifted to the blue region by $20 \mathrm{~nm}$. From a series of structural analyses, it was suggested that the fluorescence emission after smashing could be originated from the cracked surfaces. Next, the fragment crystals were ground by a mortar, creating the amorphous phase; consequently, the crystal turned into weakly orange-red luminescent powder. The broad emission band with a broad peak at approximately $605 \mathrm{~nm}$, which was shifted to the red region by $40 \mathrm{~nm}$ compared with that of the smashed crystals, was obtained. It was assumed that different molecular arrangements such as $\pi$-stacked dimers had formed after grinding, which caused the redshifted luminescence observed. The influence on the emission property of the orange crystals by increasing the hydrostatic pressure was investigated. In silicon oil, the crystals were subjected to a pressure of $5.77 \mathrm{GPa}$, and the red-shifted emission band was observed. Considering the results from the structural analyses, it was proposed that compression shortened the distance between the $\pi$-planes in the dimer, leading to red-shifted luminescence. These data propose the 
a

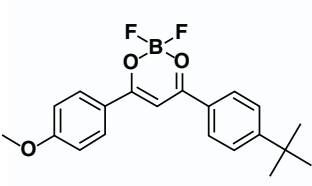

b

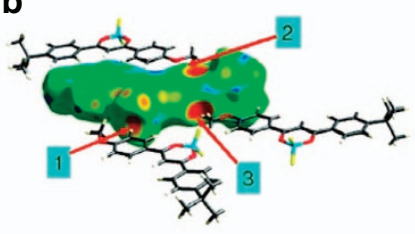

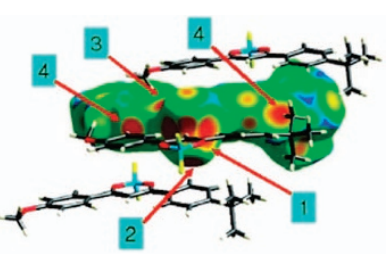

C

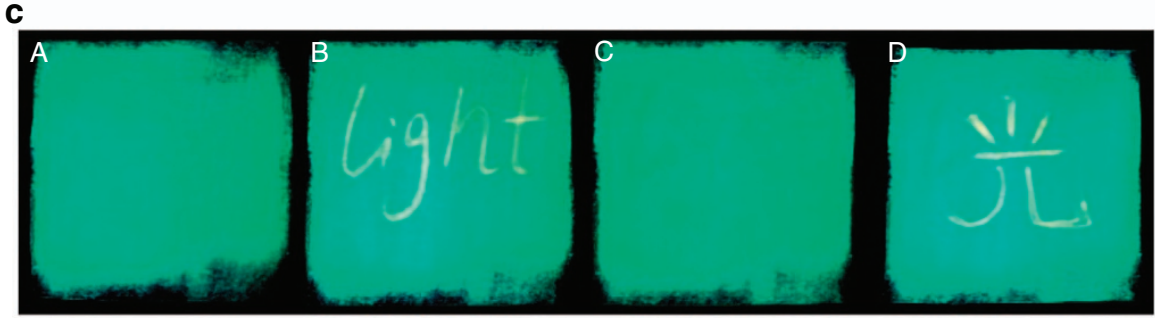

d

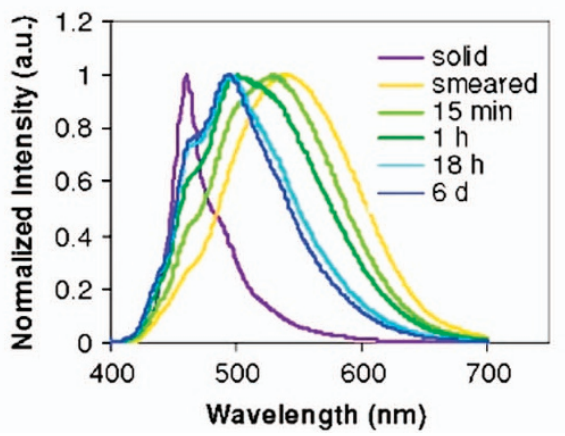

e

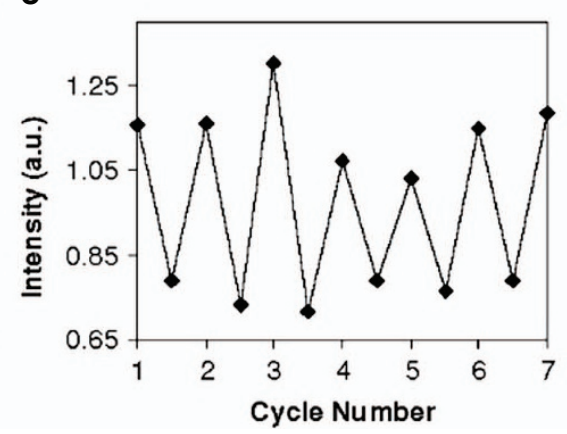

Figure 3 (a) Chemical structure of boron ketoiminate with the MFC property. (b) Crystal packing for green (left) and cyan (right) crystals and significant intermolecular interactions. (c) MFC behaviors of the complex. (d) Fluorescence emission spectra with the solid samples. (e) Fluorescence emission intensity monitored at $535 \mathrm{~nm}$ vs smearing/thermal erasing cycle number. Reprinted with permission from Maeda et al. ${ }^{37}$ Copyright 2010 American Chemical Society.

applicability of MFC boron diketonates as precise chemosensors based on the mechanical stress in a polymer or other matrices.

\section{EMISSIVE SOLID MATERIALS BASED ON THE AIE PROPERTY}

In general, the bright emissions of conjugated polymers disappear after condensation; this fact indicates that optical properties should be lost in solid or film states. These behaviors are called concentration quenching or aggregation-caused quenching (ACQ). Conversely, it has been reported that certain compounds exhibit stronger emissions in their aggregation states. Tang and colleagues first showed unique behaviors with tetraphenyl-substituted silole compounds in 2001. ${ }^{46}$ Only when the compounds were suspended in the poor solvent was a bright emission observed. This phenomenon is called AIE or AIE enhancement, and the application in highly emissive solid materials is expected to be the promising molecular design for overcoming ACQ. In particular, because ACQ directly contributes to decreases in device efficiency in the electroluminescent devices, the development of new AIE-active molecules remains of significant interest in the development of modern electric devices. In this section, we explain the recent progress of the molecular design and the development of AIE-active conjugated polymers based on organoboron complexes.

As mentioned in the Introduction section, boron diketonate has various advantages as a building block for preparing emissive materials; however, the optical properties of boron diketonate would be lost in the solid state owing to critical ACQ effect, which is similar to other fluorescent molecules. Using the ACQ molecule, the AIEactive molecule was created using boron diketonate derivatives; ${ }^{47}$ the chemistry of the resulting AIE-active organoboron complexes is described below.

Based on the proposed mechanism in the previous reports, the introduction of bulky groups into the molecules is effective at creating the AIE property. ${ }^{46}$ In the solution state, the excitation energy can be consumed by the intramolecular tumbling of these substituents, resulting in no emission being produced; conversely, these molecular motions would be suppressed in the aggregation state. Additionally, stacking and intermolecular interaction can also be disturbed by these bulky groups in the condensed state. As a result, the ACQ can be efficiently avoided, producing the solid-emissive property. To define this mechanism in the diketonate analogue, we designed and constructed the boron ketoiminate structure (Figure 5a). A comparison study between boron diketonate and related chelate complexes with regard to their electronic structures was systematically performed by Gardinier and her co-workers. ${ }^{48}$ By replacing one of the oxygen atoms with a nitrogen atom, the functional group could be incorporated. In addition, a distortion was introduced into the molecules using the relatively weaker $\mathrm{B}-\mathrm{N}$ bond compared with the $\mathrm{B}-\mathrm{O}$ bond. A series of boron ketoiminates were synthesized, and the physical properties, including the AIE behavior, were investigated.

Figure $5 \mathrm{~b}$ shows the emission spectra of ketoiminates and diketonate. Boron diketonate exhibited a strong blue emission in tetrahydrofuran (THF) $\left(5.0 \times 10^{-5} \mathrm{M}, \Phi_{\mathrm{PL}}=0.91\right)$, and ACQ occurred in the aggregation state $\left(\Phi_{\mathrm{PL}}=0.36\right)$; conversely, ketoiminates showed very weak emissions in the THF solutions $\left(5.0 \times 10^{-5} \mathrm{M}, \Phi_{\mathrm{PL}} \leqslant 0.01\right)$. This result should be caused by the energy consumption via the 

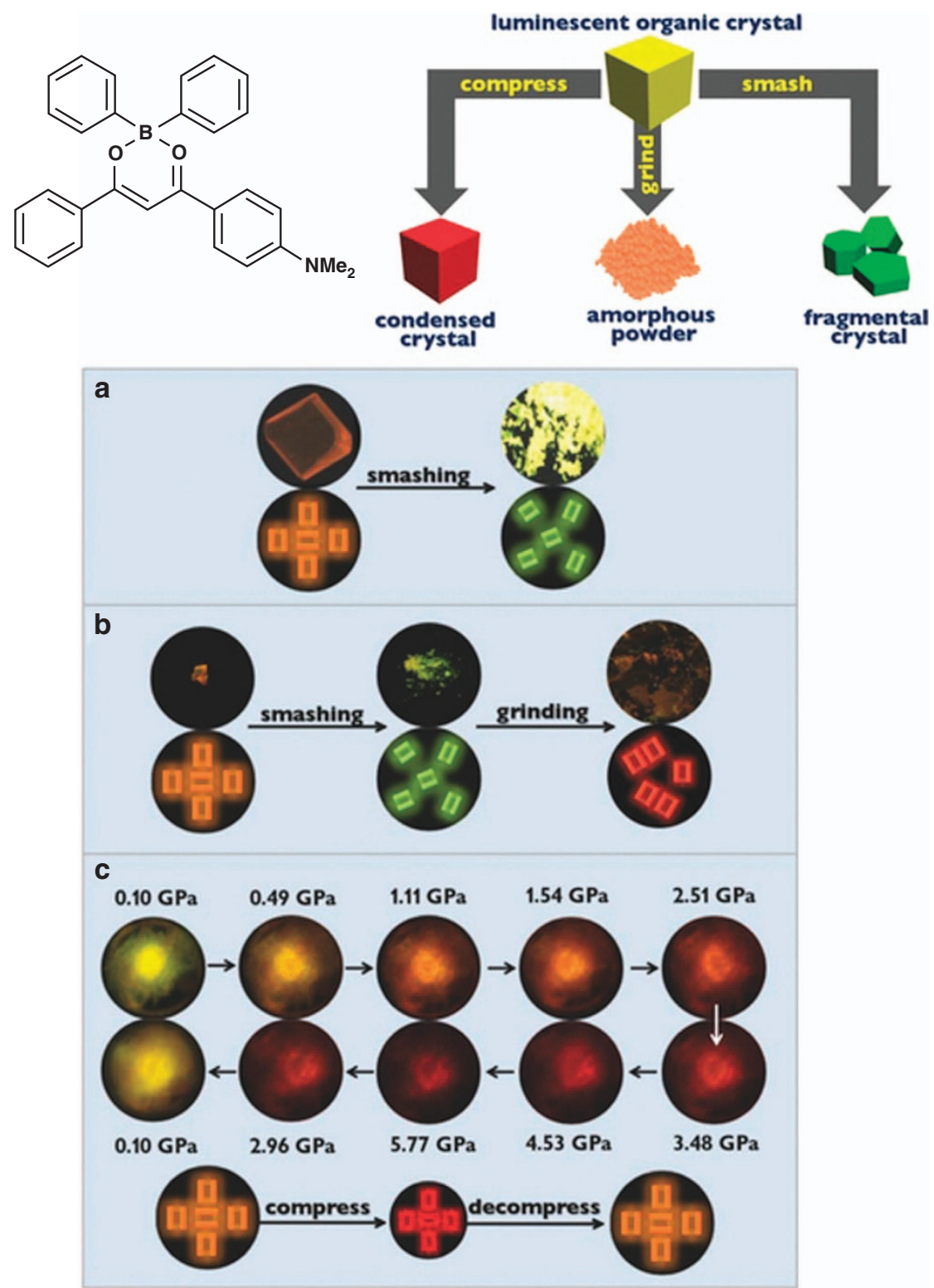

Figure 4 Photographic images and molecular arrangement transformation of the orange crystals before and after smashing (a), fragmental crystals before and after grinding (b) and orange crystals under different isotropic pressures (c). Reprinted with permission from Kumar et al. ${ }^{38}$ Copyright 2015 Wiley-VCH Verlag $\mathrm{GmbH}$ and Co. KGaA.

intramolecular motions in the ketoiminate ring. Notably, the aggregation states of ketoiminates in the mixed solvent of $\mathrm{THF} / \mathrm{H}_{2} \mathrm{O}(1 / 9$ $(\mathrm{v} / \mathrm{v}))$ showed significant increases in their emission intensities $\left(\Phi_{\mathrm{PL}}=\right.$ $0.30-0.76$ ). These results clearly indicate that ketoiminates are AIEactive molecules. The six-membered ring, which included a $\mathrm{B}-\mathrm{N}$ bond, should have an important role in developing AIE properties.

The AIE behaviors of boron ketoiminates can be significantly increased by the crystal packing structures present. Based on X-ray single-crystal analyses, it was shown that the molecular structure of diketonate was highly planar, while ketoiminates included asymmetric structures and particularly distortion in the complex that originated from relatively longer bond lengths of $\mathrm{B}-\mathrm{N} .{ }^{48}$ These data indicate that the boron-chelating rings on ketoiminates should be more flexible than those on diketonates; therefore, consumption of excitation energy by molecular tumblings should occur in the solution state. As a result, a small emission was observed from the solutions containing ketoiminates. Conversely, these molecular tumblings, which induced the non-radiative decay, should be suppressed in the aggregation state. Because of the steric hindrance of the substituted nitrogen atom, intermolecular interaction and stacking could be disturbed, resulting in the inhibition of the ACQ; thus, AIE behavior could be obtained. As mentioned later, similar data were obtained from the X-ray crystal analyses and from the optical measurements in other studies of boron diiminates. Thus the above mechanism is the most likely explanation on the AIE behavior of boron ketoiminates and diiminates.

The MFC response was also found in AIE-active boron ketoiminate derivatives (Figure 6a). ${ }^{49} \mathrm{~A}$ variety of boron ketoiminates with diverse substituent groups at both ends were synthesized, and the substituent effects on the optical properties were investigated. Similar to above, boron ketoiminates exhibited AIE properties. In addition, the MFC 
a<smiles>COc1ccc(C2=CC(c3ccc(OC)cc3)=C(F)B(F)O2)cc1</smiles>

(DK)

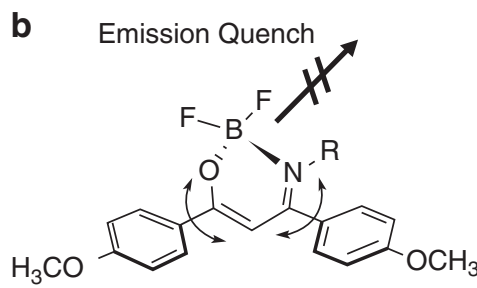

In Solution

C

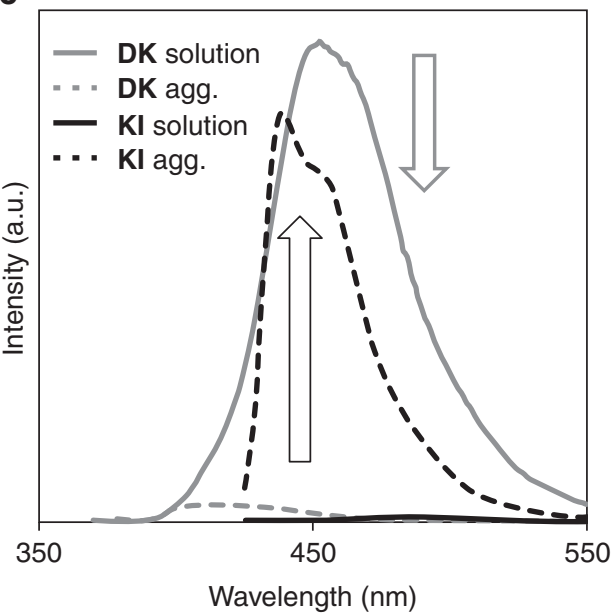

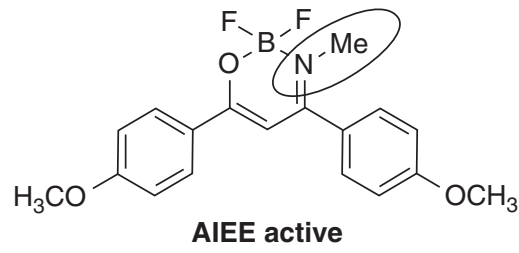

AIEE active

(KI)

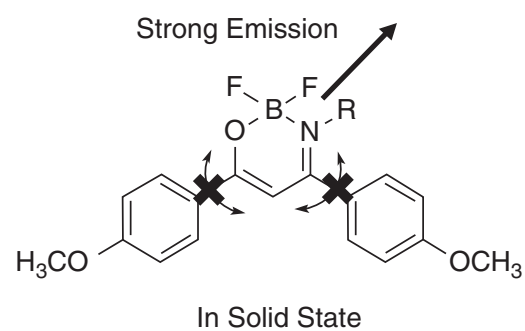

d

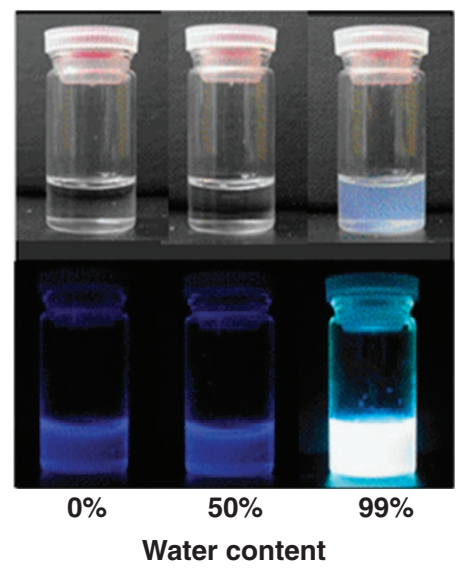

Figure 5 (a) Chemical structures of ACQ diketonate and AlE-active ketoiminate complexes. (b) Plausible models of emission property of boron ketoiminate. (c) Emission spectra of the complexes. Aggregation was induced by adding water (99 vol\%) to the THF solutions. (d) Pictures of the samples by increasing the water contents in the media. Reprinted with permission from Perumal et al. ${ }^{40}$ Copyright 2013 Wiley-VCH Verlag GmbH and Co. KGaA.

behaviors were observed. Interestingly, the hypsochromic and bathochromic shifts of the emission bands were individually observed from boron ketoiminates depending on the chemical structures of the end groups present (Figure 6b). Based on the structural and thermal analyses, it was revealed that the MFC property of boron ketoiminates should be derived from a phase transition between the crystalline and amorphous states. In particular, the direction of the peak shifts of the emission bands was controlled by the degree of the steric hindrance of the end group: from the complexes with relatively small end groups, the AIE colors were red at the initial crystalline state; after grinding, hypsochromic changes were induced; conversely, the complexes with larger end groups presented the yellow AIE before grinding; and after adding the mechanical stimuli, bathochromic shifts in the emission spectra were detected. These changes were explained as the alteration of the degree of intermolecular interaction between the crystalline and amorphous states (Figure 6c). Boron ketoiminates with small substituents formed relatively densely packed structures in crystalline states owing to the small steric hindrance present. The closed packing structure in the crystalline state can induce high planarity and strong $\pi-\pi$ intermolecular interactions relative to those in the amorphous state. As a result, the emission band could be shifted to shorter wavelengths after transformation to the amorphous state. Conversely, the dense packing structure could be disturbed by the large substituents in the crystalline states, resulting in the formation of loose packing even in the crystalline state. Beccause of the mechanical stress, the molecules could form random configuration, and stronger $\pi-\pi$ intermolecular interactions relative to the crystalline samples could be obtained in the amorphous states. Chloro-, bromo- and iodosubstituted boron ketoiminates showed larger emission shifts than those of other materials. The intermolecular halogen bond could influence these large, anomalous emission shifts.

By expanding the conjugation systems with fused heterocycles involving the nitrogen atom in the ketoiminate complexes, various AIE-active molecules can be obtained. Kubota et al. ${ }^{50}$ presented AIE-active molecules and a complex with the same degree of fluorescence quantum yields in both the solution and solid states. Sun and colleagues reported the creation of quinoxaline-fused complexes. Although ACQ was observed from the solid samples, the 
a

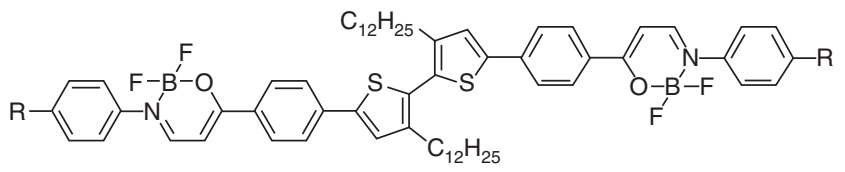

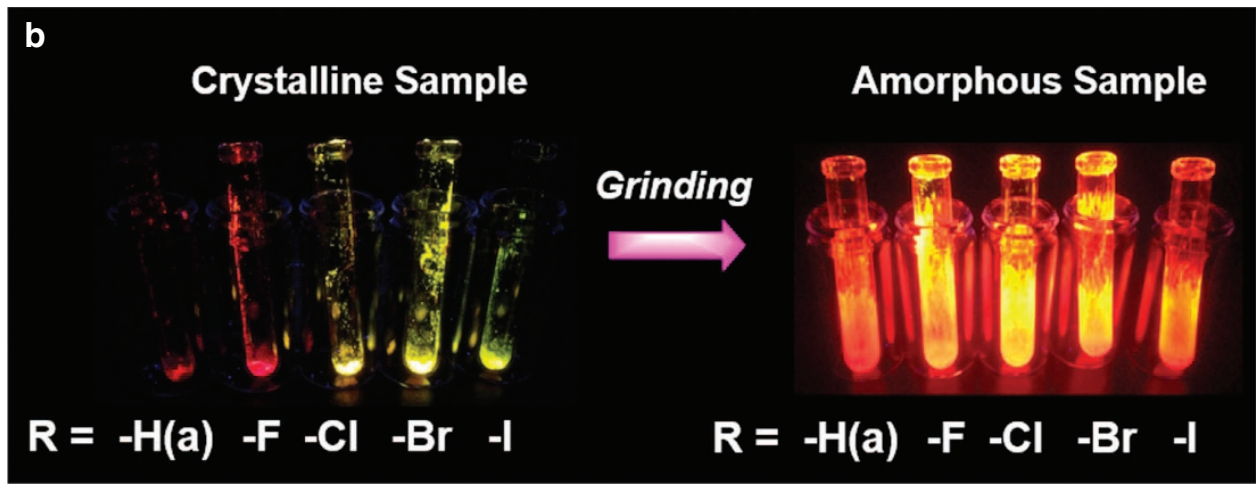

C

In the case of large substituent

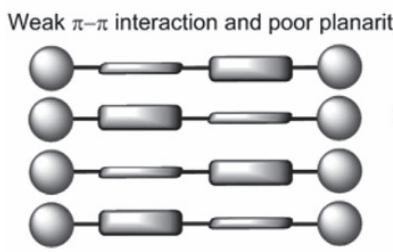

In the crystalline state
Increase in $\pi-\pi$ interaction and planarity

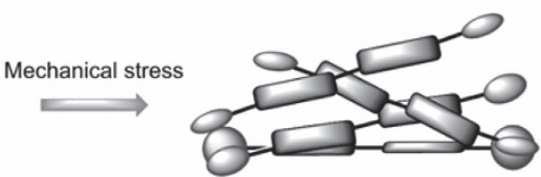

In the amorphous state

In the case of small substituent

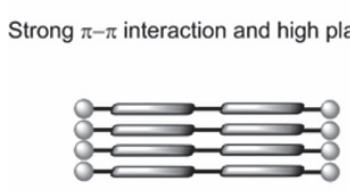

In the crystalline state

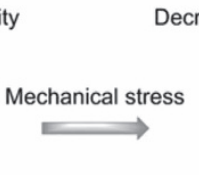

Decrease in $\pi-\pi$ interaction and planarity

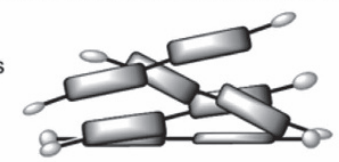

In the amorphous state

Figure 6 (a) Chemical structures of boron ketoiminates with the AIE and MFC properties. (b) Photograph of the boron ketoiminates in the crystalline and amorphous states under UV irradiation. (c) Speculated mechanisms of the mechanofluorochromism depended on the end groups. Reprinted with permission from Zhang et al. ${ }^{42}$ Copyright 2015 Wiley-VCH Verlag GmbH and Co. KGaA.

emission properties of the material were determined; ${ }^{51}$ it was demonstrated that the emission intensity could be tuned reversibly by fuming the material with trifluoroacetic acid (TFA) and triethylamine (TEA) vapors. Pyridine-fused complexes were also synthesized, and their AIE properties were clarified. ${ }^{52}$ These materials are expected to be a practical platform for emissive devices and chemical sensors.

To create MFC behavior with AIE-active molecules, the nitrogen must be replaced in the above systems. More recently, Šket and colleagues created a multi-functional solid-emissive complex based on boron diketonate (Figure 7). ${ }^{53}$ It was found that, only by introducing two methoxy groups into one of the phenyl rings at meta positions, boron diketonate could be added to create numerous chromic effects, including mechano-chromism, thermo-chromism and chronochromism, in the solid state. This molecule can form two polymorphs with different mutual orientations of the two methoxy groups. In one of the polymorphs, the crystal packing structure was stabilized with intermolecular hydrogen bonds. Various chromic effects and crystallization-induced emission enhancement could be observed by the perturbation of the packing structure. Additionally, self-assembling crystalline plate-like microstructures or microfibers that create an significant optical waveguide effect were also demonstrated. This type of sophisticated boron diketonate exemplifies the versatility of this material as an optical functional material.

\section{CRYSTALLIZATION-INDUCED EMISSION FROM BORON DIIMINATES}

By replacing one of the oxygen atoms with nitrogen in diketonate, AIE properties were obtained. Next, the properties of boron diiminates are explained. Compared with boron diketonates and boron ketoiminates, there is room in boron diiminates for functionalization via their two nitrogen atoms, which can bond to various substituents (Scheme 1). Because the regulation of intermolecular interactions in solid state is important for strong emissions, boron diiminates with high functionalities have a high potential for use as AIE-active materials. 
The optical properties of diiminates were investigated. ${ }^{54}$ Similar to ketoiminates, diiminates also exhibited AIE properties. The emission spectra showed marginal luminescence in THF $(\Phi<0.01)$, while crystallization led to large increases in the emission intensities; these results clearly indicate that synthesized boron diiminates are AIE-active molecules. Next, the modulation of the optical properties was evaluated in the solid states. Two types of solid samples were prepared in the amorphous and crystalline states. In the emission spectra, strong emissions of diiminate DI was observed from the crystalline samples (Figure 8). Conversely, the amorphous samples presented weaker emissions. It should be emphasized that the crystallization induced approximately 15-fold larger emission<smiles>COc1cc(OC)cc(C(=O)/C=C(/OB(F)F)c2ccccc2)c1</smiles>

Mechanochromism

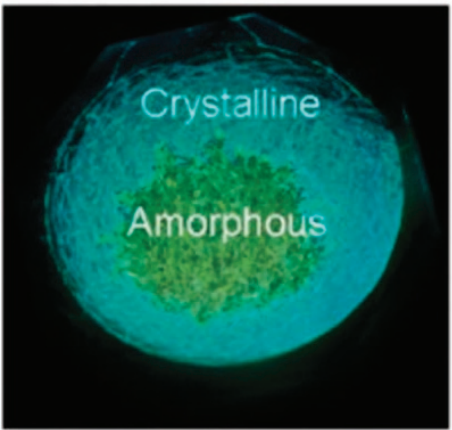

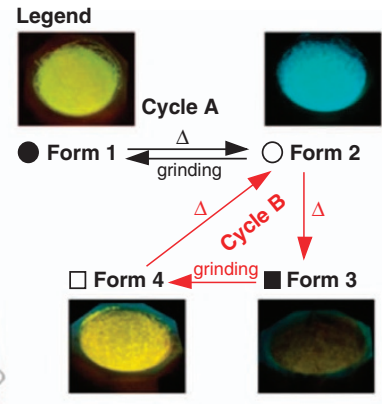

Aggregation-Induced Emission
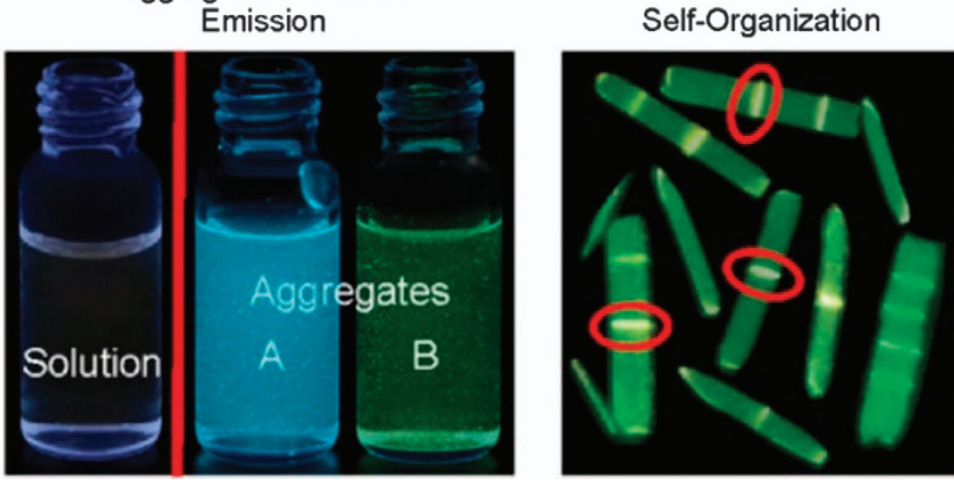

Figure 7 Boron diketonate with numerous chromic properties. Reprinted with permission from Luo et al. ${ }^{46}$ Copyright 2015 American Chemical Society.
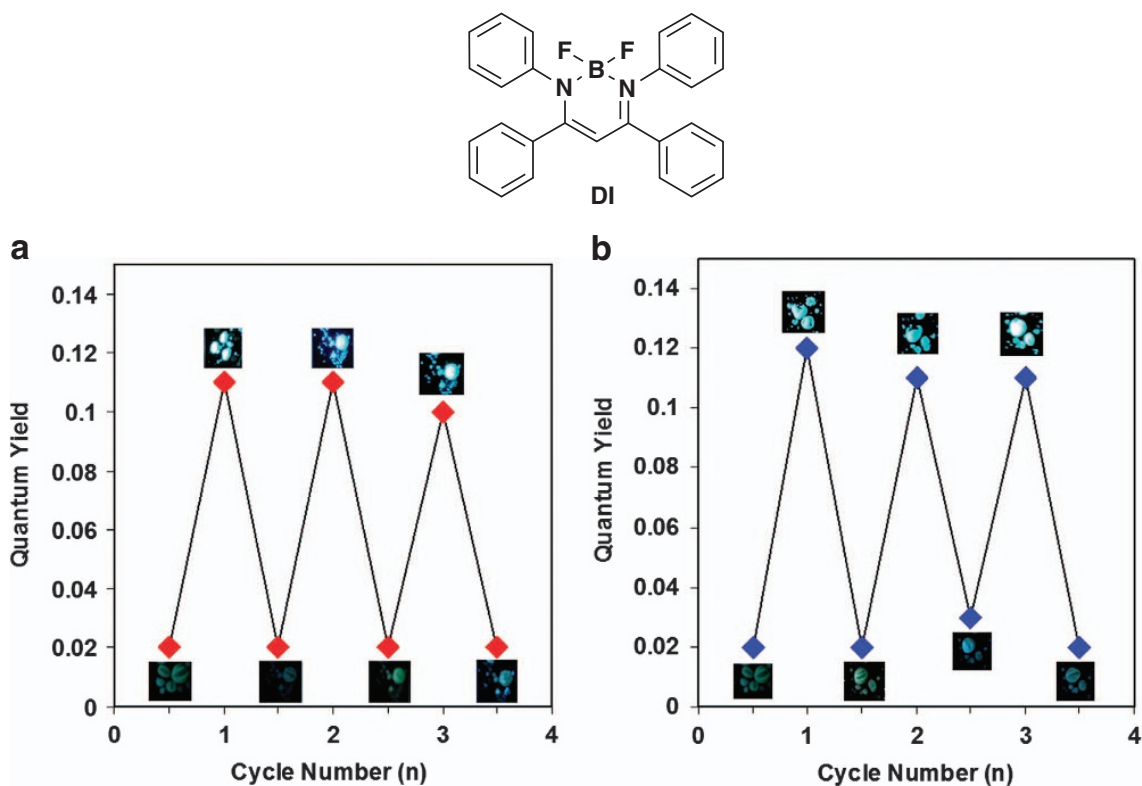

Figure 8 Repeated switching of the emission of DI between amorphous and crystalline states by (a) fuming-heating and (b) heating-cooling cycles upon. Reprinted with permission from Yoshii et al. ${ }^{47}$ Copyright 2014 Wiley-VCH Verlag GmbH and Co. KGaA. 
efficiencies from the amorphous state $\left(\Phi_{\mathrm{PL}, \text { crystal }}=0.59\right.$, $\left.\Phi_{\mathrm{PL} \text {,amorphous }}=0.04\right)$ using the diiminate derivative. From these results, it is clearly shown that boron diiminates are crystallizationinduced emission-active units. The colors of the crystallizationinduced emission can also be modulated from blue to red by the substituents used.

\section{EMISSIVE POLYMERS BASED ON BORON DIKETONATES}

Conjugated polymers have attracted attention as platforms for fabricating organic optical and/or electronic devices owing to their many advantages, such as good film formability, solubility in ordinal solvents, stability with regard to environmental changes and emission properties in the film states. The various properties of conjugated polymers can be modulated via combination with functional units. For example, emission colors can be significantly altered by introducing functional units into the adjacent position via $\pi$-conjugation to form charge transfer (CT) states. By modulating the electron acceptors in the main polymer chains, strong emissions were observed from visible to near-infrared regions. As another example, by changing the feed ratios of each co-monomer, a series of functional optical polymers were synthesized with various ratios of chromophores in the primary polymer chains. ${ }^{55}$ These polymers showed useful characteristics such as multiple-color materials and white-light emission. Additionally, conjugated polymers containing organoboron compounds have been recognized as efficient charge transporters and optical functional materials. ${ }^{56}$ Thus, not only the construction of conjugation systems but also the modulation of electronic structures are important to obtain highly functionalized materials. In this section, a series of polymers composed of boron diketonates and their derivatives and their optical properties are explained.
By modulating the localization of the electronic orbitals, the emission properties of a material can be varied. Diarylboron diketonates were synthesized, and their electronic structures were characterized with experimental and theoretical investigations (Figure 9). ${ }^{57}$ In the experimental results, it was shown that the electronic structures of boron atoms significantly affect their emission properties. The boron complex with strong electron-withdrawing $\mathrm{C}_{6} \mathrm{~F}_{5}$ groups presented high fluorescence. Conversely, no emission was observed from the diketonate complex with diphenyl groups. It was suggested that the highest occupied molecular orbital (HOMO) of the diphenyl complex should not be localized on the diketonate moiety. Based on these findings and the results from computer modeling, it was presumed that the emission of diphenyl diketonate moiety could be restored by extending conjugation along the polymer linker, and the HOMO of diphenylboron moiety should be delocalized on the entire polymeric diketone ligand. To prove the validity of this idea, the organoboron polymers with 1,3-diketonate structure at the main chain were prepared. ${ }^{58}$ The peak maxima in the absorption and emission spectra were shifted to longer wavelengths by coordinating the boron atoms on the polymeric ligands in the solution states. In addition, polymers with diphenyl and pentafluorodiphenyl groups attached on their boron atoms emitted orange and red light, respectively. The conjugation system was extended by introducing polymeric ligands, leading to strong fluorescence emissions.

Facile methodology based on polymer reactions to regulate the electronic structures of conjugated polymers was demonstrated for optimizing the device properties and obtaining diverse optical materials. ${ }^{59}$ Alternating copolymers composed of $\beta$-diketonate and fluorene in the primary chains were synthesized. Then, using the synthesized polymers as a ligand, the boron-containing polymers in

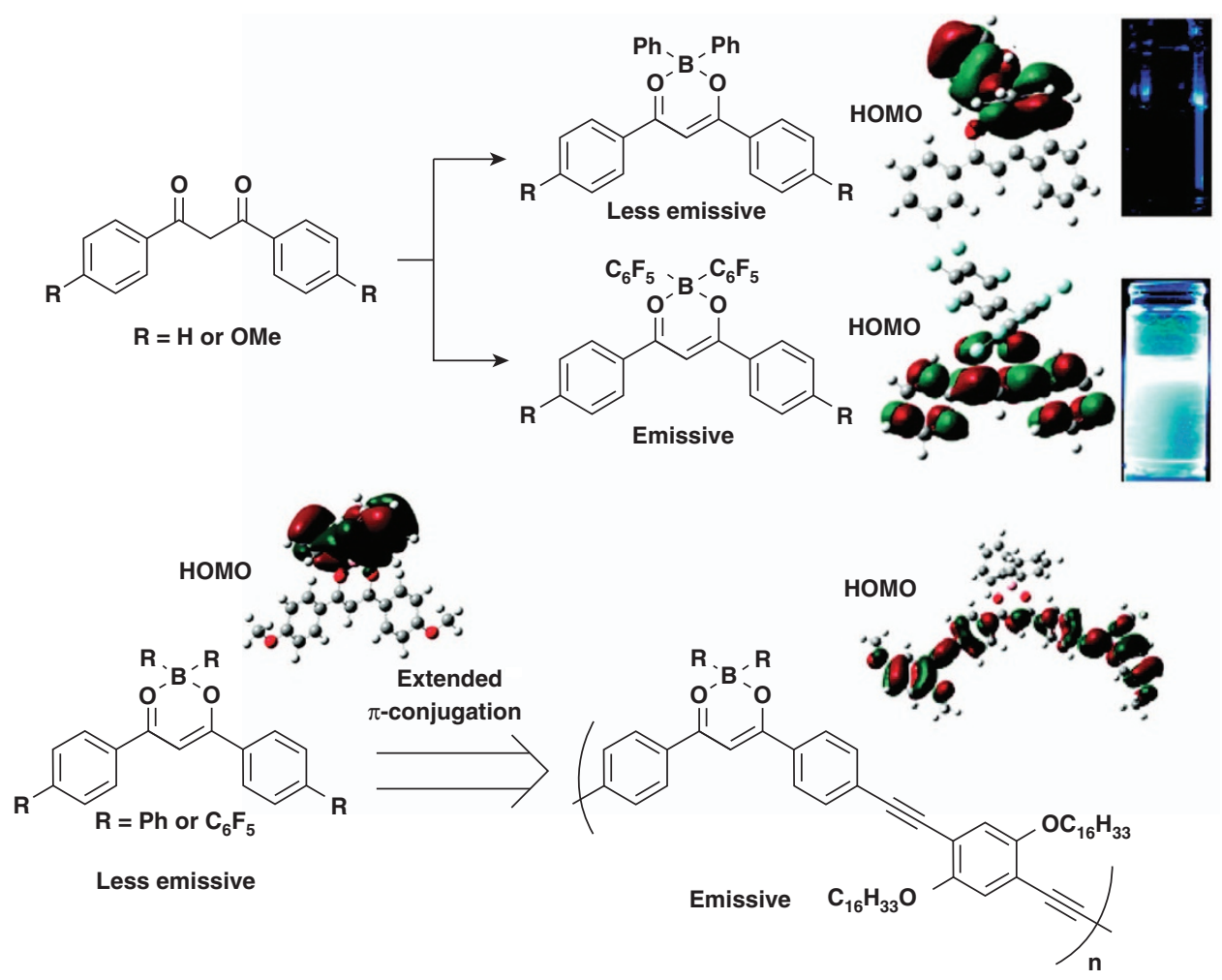

Figure 9 Localization of HOMOs and emission properties of the boron diketonates. Reprinted with permission from Kubota et al. ${ }^{50}$ Copyright 2008 American Chemical Society. 
which $21,41,58,71$ and $100 \%$ of the diketonate moiety were occupied by boron were prepared in the postmodification, respectively. The electronic properties of the series of boron diketonate-containing polymers were examined. It was observed that the emission bands in the longer wavelengths were observed by increasing the boron introduction rate. The strong electron coupling between fluorene and boron diketonate could provide significant emission bands. These data indicate that the band gap energy can be controlled by boron complexation in the conjugated polymer. This tunable functionality can be used to adjust energy levels for improving the conversion efficiencies in electroluminescence devices and photovoltaic cells.

\section{AIE PROPERTIES OF KETOIMINATE-CONTAINING POLYMERS}

Color tuning of the emissions was also demonstrated with the boron ketoiminate-containing polymers (Figure 10). ${ }^{60}$ A series of alternating copolymers involving boron ketoiminate units were prepared with fluorene and bithiophene co-monomers. It was observed that the polymers exhibited strong emissions in the solution $\left(\Phi_{\mathrm{em}}=0.46\right.$ to 0.80) owing to the introduction of the boron ketoiminates into the

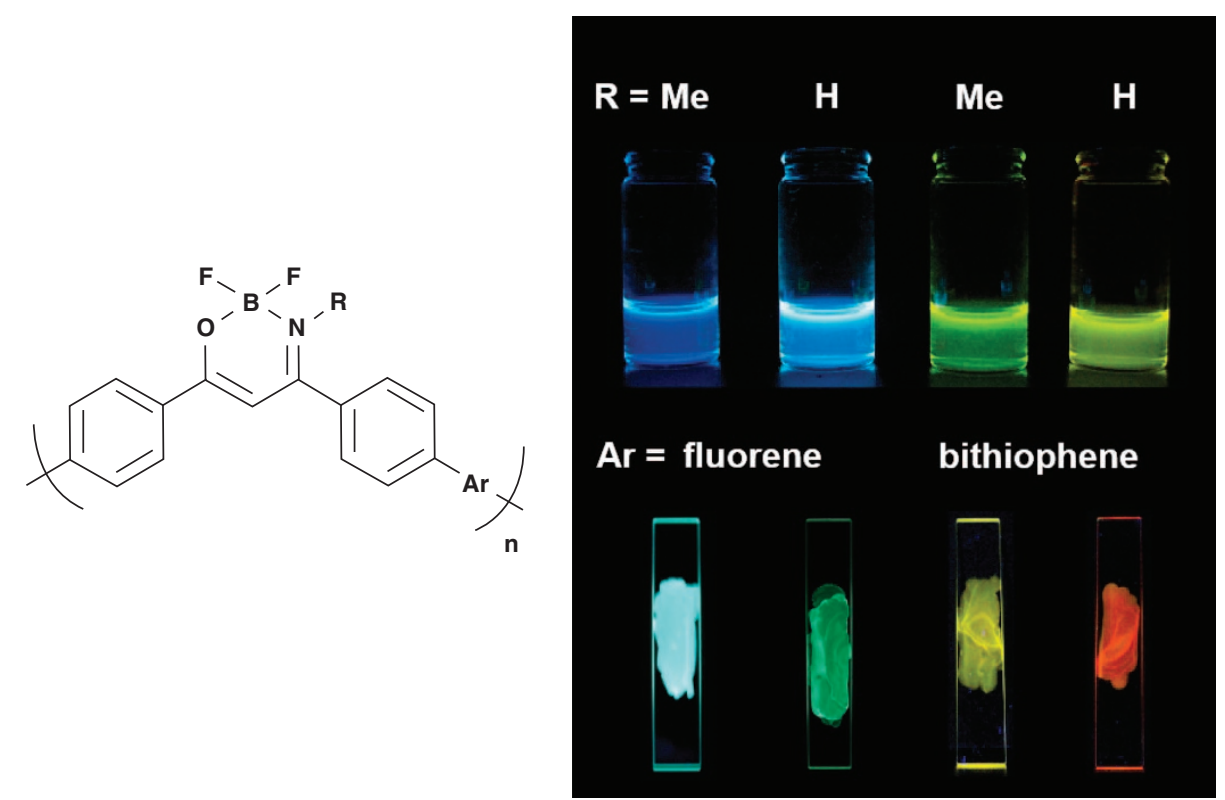

Figure 10 Chemical structures and emission properties of the polymers containing boron ketoiminate. Reprinted with permission from Galer et al. ${ }^{53}$ Copyright 2014 Wiley-VCH Verlag GmbH and Co. KGaA.

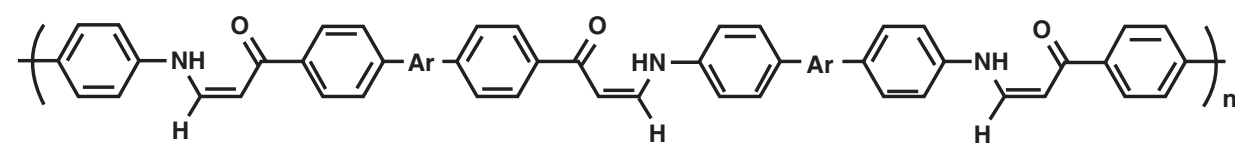

FLP, BIP

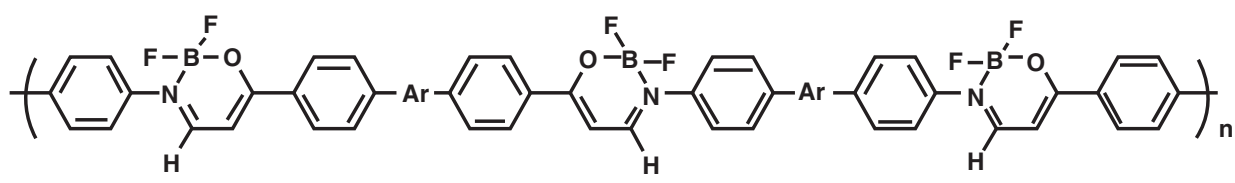

LBP, BIBP

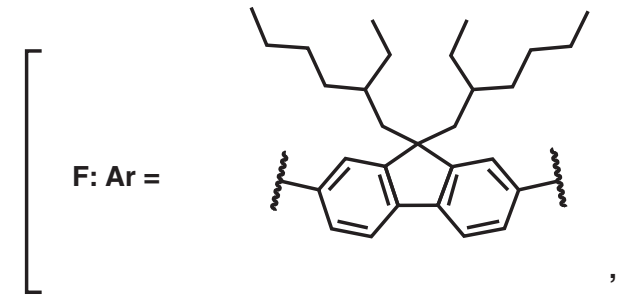

$\mathrm{T}: \mathrm{Ar}=$

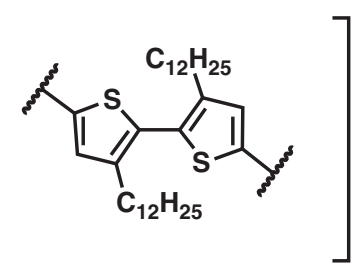

Scheme 4 Chemical structures of ketoiminate polymers. 
primary polymer chains. Their optical properties were explained by the donor-acceptor interaction between the boron ketoiminate as an acceptor unit and the electron-donating co-monomer, such as fluorene or bithiophene. Additionally, even in the solid states, these polymers showed bright emissions $\left(\Phi_{\mathrm{em}}=0.13-0.38\right)$, and their emission colors could be tuned from blue to orange by substituting nitrogen atoms. Using these materials as emissive film materials is thus possible.

Next, based on boron ketoiminate, the main-chain-type conjugated polymers were synthesized to create polymeric AIE-active materials. ${ }^{61}$ The series of boron ketoiminates shown in Scheme 4 was designed. Ketoimine derivatives can form three possible tautomeric structures: ketoimine, enolimine, and enaminoketone forms (Scheme 5). It is known that the enaminoketone form has the highest thermal stability of three tautomers. Via boron complexation, the structures of the ketoiminates would be transformed into a similar electronic structure

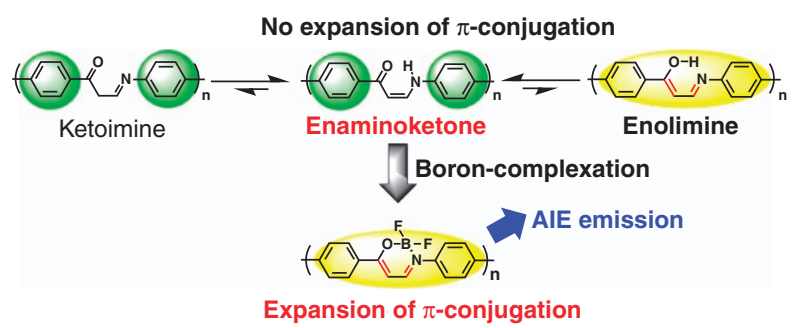

Scheme 5 Tautomerization of ketoimines and immobilization at the enaminoketone form by boron complexation. to the enolimine form; thus it can be said that the tautomerization of ketoimine can be induced by the introduction of boron. Additionally, it is presumed that boron-induced tautomerization could change the electronic interaction mediated through the ketoimine moiety. Finally, boron complexation at the ketoimine skeleton can induce the extension of the conjugated system in the main polymer chain. Thus a significant possibility to obtain AIE-active materials with tunable optical properties based on boron ketoiminate materials exists.

From the emission spectra, it was found that the boron ketoiminate-containing polymers showed significantly higher quantum yields in the solid states (FLBP: $\Phi_{\mathrm{PL}}=0.13$, BIBP: $\Phi_{\mathrm{PL}}=0.06$ ) than those in THF (FLBP: $\Phi_{\mathrm{PL}}=0.10$, BIBP: $\Phi_{\mathrm{PL}}=0.04$ ), as shown in Figure 11. These results clearly indicate that the synthesized polymers should be AIE-active polymers. Conversely, without boron complexation, the typical ACQ behaviors were obtained from the polymers. In THF, relatively higher quantum yields were observed (FLP: $\Phi_{\mathrm{PL}}=0.01$, BIP: $\left.\Phi_{\mathrm{PL}}=0.09\right)$, while the solid sample showed decreases in the quantum yields (FLP: $\Phi_{\mathrm{PL}}<0.01$, BIP: $\Phi_{\mathrm{PL}}=0.02$ ). This fact indicates that boron complexation of the ketoimine units is responsible for the expression of AIE properties. By changing the co-monomer unit, the AIE color was altered. FLBP exhibited an emission band with a peak at $562 \mathrm{~nm}$ (that is, yellow). Conversely, BIBP exhibited a red-color emission $\left(\lambda_{\mathrm{em}}=683 \mathrm{~nm}\right)$. The electron donating-accepting interaction should occur between boron ketoiminate and bithiophene units through the primary polymer chains. This result indicates that the optical properties of this material can be tuned by varying the molecular design. To demonstrate the AIE behaviors of the boron ketoiminate polymers, the dependency of the emission properties on the solvent compositions was examined in the $\mathrm{THF} / \mathrm{H}_{2} \mathrm{O}$ solvent
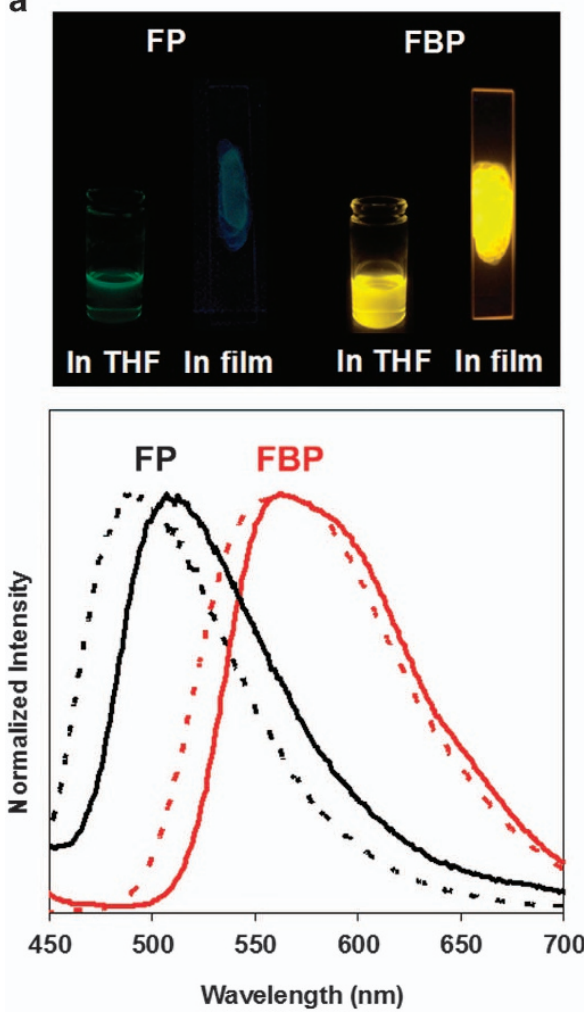
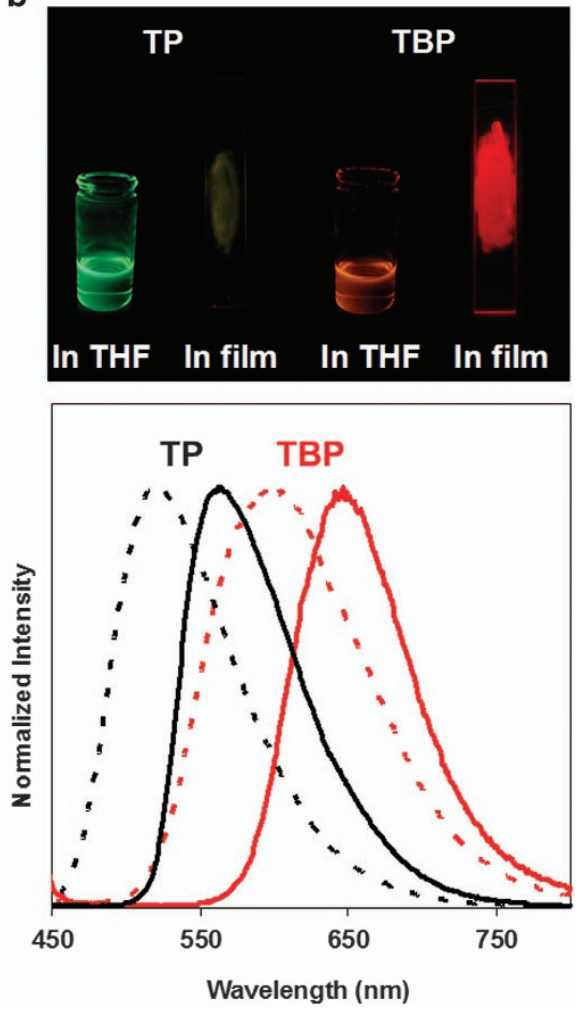

Figure 11 Photographs and emission spectra of (a) fluorene-based polymers and (b) bithiophene-based polymers in THF and solid states under UV irradiation. Reprinted with permission from Yoshii et al. ${ }^{54}$ Copyright 2014 American Chemical Society. 

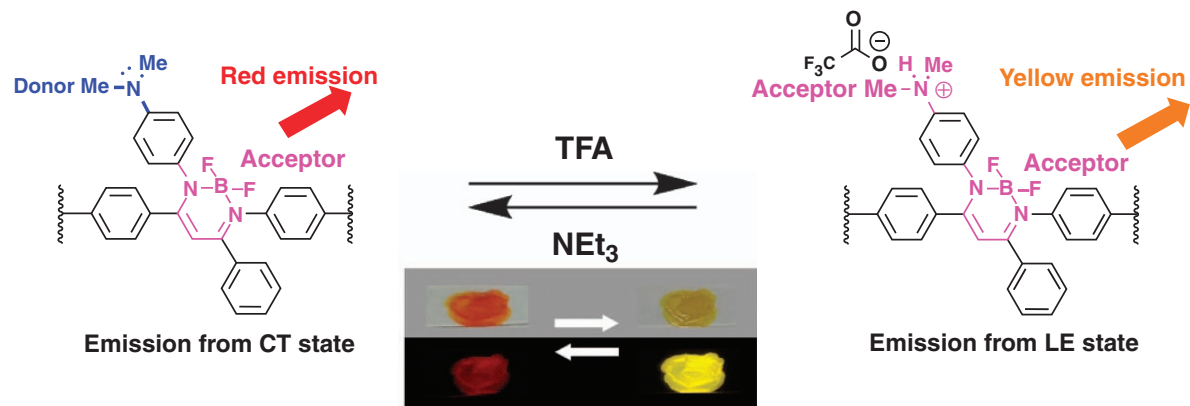

Scheme $6 \mathrm{pH}$-responsive behavior of the diiminate polymer and a photograph of $\mathrm{pH}$ sensing. Reproduced with permission from Nagai et al. ${ }^{57}$ Copyright 2014 American Chemical Society.

mixture system. The enhancement of the emission intensities was observed by increasing the water content $\left(\mathrm{H}_{2} \mathrm{O} \geqslant 90\right.$ vol\%). The aggregation of the polymers should improve the emission. Thus these results show that AIE-active polymers with the tunable color emissions were created. The introduction of boron can also induce structural torsion in the conjugated systems; as a result, the ACQ effect might be avoided in the film state.

By modifying the nitrogen atom in boron ketoiminate complexes with substituents, additional functions can be introduced. ${ }^{62}$ Boron ketoiminate-containing polymers with the chiral group at the nitrogen atom were synthesized. These polymers showed circular dichroism and CPL properties; in particular, the large CPL dissymmetry factors were exhibited by the polymers $\left(g_{\text {lum }}=+0.349\right.$ and +0.105$)$. The strong CPL observed could be attributed to the amplification effect owing to the conjugated polymer structure.

Boron ketoiminate-containing polymers were recently applied to the field of cell imaging. ${ }^{63}$ A series of conjugated polymers with various substituents at the phenyl group on the nitrogen atom in boron ketoiminate complexes were created. The synthesized polymers showed AIE properties with different colors depending on the substituents. Highly emissive nanoparticles can be obtained with the polymers; these materials have low cytotoxicity and high photostability and can thus be used as probes for HeLa cell imaging. By regulating biodistribution, and adding the stimuli-responsiveness, these materials could be applied in sensing tools for surveying various biological events or reactions.

\section{BORON DIIMINATE-CONTAINING POLYMERS AND THEIR APPLICATIONS FOR FILM-TYPE CHEMOSENSORS}

The electron-accepting ability of boron contributes to the enhancement of the electronic interactions to the neighboring electrondonating unit. Thus, by modulating the electron-donating and/or electron-accepting abilities of the components in the conjugated polymers, the electronic structures could be significantly altered, leading to significant changes in their optical properties. Thus it is proposed that the AIE-active conjugated polymers should be a suitable scaffold for fabricating film-type emissive chemosensors. Diiminatecontaining polymers also exhibited AIE properties. ${ }^{64}$ By changing the co-monomer unit, certain optical properties such as color can be tuned. Based on these polymers, the dynamic modulation of the optical properties was performed by acid- and base-sensing experiments (Scheme 6). After exposure to TFA and TEA vapors, the changes in the emission properties were monitored with the film samples. Thus the AIE properties of boron diiminates-containing polymers were shown to dynamically change. When the film was placed in a small container that was saturated with TFA vapors, the

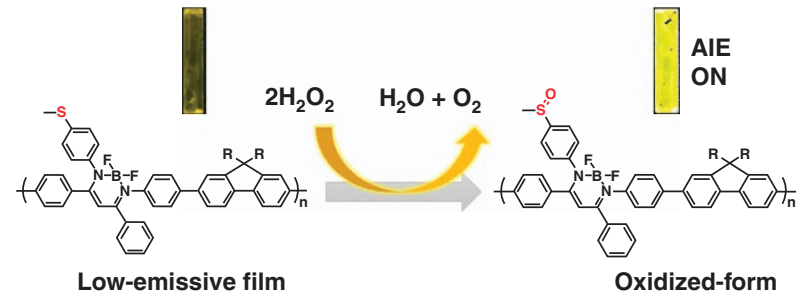

Scheme 7 Schematic illustration of $\mathrm{H}_{2} \mathrm{O}_{2}$ detection with a film-type sensor with the oxidation-induced AIE property. Reproduced with permission from Nagai et al. ${ }^{58}$ Copyright 2015 The Royal Society of Chemistry.

fumed film exhibited a blue-shifted emission spectrum compared with those of the unaltered sample; the emission color was changed from red to yellow. Additionally, the optical properties of the TFA-exposed film were converted back into the parent state after exposure to TEA vapor. These data clearly show that the optical properties of these materials can be tuned reversibly. The strong donor-acceptor interaction between the amino group and the boron-chelating ring could be suppressed by the protonation of the amino group; as a result, the color was changed. Boron diiminate-containing polymers are thus promising for application in various types of film-type optical sensors.

To extend this idea regarding the application of AIE-active conjugated polymers as film-type optical sensors, another system was constructed. Films with the AIE-active copolymers composed of fluorenes and sulfide-substituted boron diiminates were prepared, and the changes in their optical properties via oxidation were evaluated (Scheme 7). ${ }^{65}$ Hydrogen peroxide $\left(\mathrm{H}_{2} \mathrm{O}_{2}\right)$ is classified as a reactive oxygen species and is endogenously produced during energy metabolism. Excessive $\mathrm{H}_{2} \mathrm{O}_{2}$ induces cell damage, leading to many pathological problems, such as diabetes, cardiovascular diseases and cancer; thus the development of facile detection techniques is required. By soaking the film samples of the polymer in the solution containing $\mathrm{H}_{2} \mathrm{O}_{2}$, the emission intensity from the film gradually increased during incubation. The quantum yields were correspondingly enhanced up to three times via oxidation at the sulfide groups. This could be attributed to the facilitated, charge-transfer, emissive fluorescence between the fluorene units and the boron diiminates with the enhanced electron-withdrawing ability by replacing sulfide with sulfoxide groups. These data indicate that the synthesized polymer can work as a film-type optical sensor with turn-on fluorescence. Thus it is proposed that AIE-active polymeric materials should be a suitable platform as bioprobes for longitudinal monitoring of bio-related reactions. 


\section{CONCLUSION}

The optical properties and applications of boron $\beta$-diketonates and related compounds, including polymers, are the focus of this review. Although boron diketonates were identified several decades ago and have simple structures, various optical functions have been reported during the past decade, as described in this review. In addition, because of the ease of synthetically constructing boron diketonate derivatives, it is relatively easy to conjugate the material with other functional units to obtain different combinations of functions. Recently, the development of organic electronic devices has become a critical issue for using advanced functions and for reducing environmental burden. To satisfy the demands and requirements of the modern material designs, versatile building blocks are necessary, and boron diketonates seem to be valid building blocks for creating new functional materials that have currently unknown functions and that have never been seen before.

\section{CONFLICT OF INTEREST}

The authors declare no conflict of interest.

1 Chujo, Y. \& Tanaka, K. New polymeric materials based on element-blocks. Bull. Chem. Soc. Jpn 88, 633-643 (2015).

2 Matsumi, N., Naka, K. \& Chujo, Y. Extension of $\pi$-conjugation length via the vacant p-orbital of the boron atom. Synthesis of novel electron deficient $\pi$-conjugated systems by hydroboration polymerization and their blue light emission. J. Am. Chem. Soc. 120 5112-5113 (1998)

3 Sun, C., Hudson, Z. M., Helander, M. G., Lu, Z.-H. \& Wang, S. A polyboryl functionalized triazine as an electron transport material for OLEDs. Organometallics 30 5552-5555 (2011).

4 Braunschweig, H. \& Kupfer, T. Recent developments in the chemistry of antiaromatic boroles. Chem. Commun. 47, 10903-10914 (2011).

5 Ulrich, G., Ziessel, R. \& Harriman, A. The chemistry of fluorescent BODIPY dyes: versatility unsurpassed. Angew. Chem. Int. Ed. 47, 1184-1201 (2008).

6 Rao, Y.-L., Amarne, H. \& Wang, S. Photochromic four-coordinate N,C-chelate boron compounds. Coord. Chem. Rev. 256, 759-770 (2012).

7 Zhang, X., Cui, M., Zhou, R., Chen, C. \& Zhang, G. Facile synthesis of $\beta$-diketone alcohols for combined functionality: initiation, catalysis, and luminescence. Macromol. Rapid Commun. 35, 566-573 (2014)

8 Chow, Y. L., Johansson, C. I., Zhang, Y.-H., Gautron, R., Yang, L., Rassat, A. \& Yang, S.-Z. Spectroscopic and electrochemical properties of 1,3-diketonatoboron derivatives. J. Phys. Org. Chem. 9, 7-16 (1996)

9 Kajiwara, Y., Nagai, A., Tanaka, K. \& Chujo, Y. Efficient simultaneous emission from RGB-emitting organoboron dyes incorporated into organic-inorganic hybrids and preparation of white light-emitting materials. J. Mater. Chem. C 1, 4437-4444 (2013).

10 Okamoto, A., Tainaka, K., Nishiza, K.-I. \& Saito, I. Monitoring DNA structures by dua fluorescence of pyrene derivatives. J. Am. Chem. Soc. 127, 13128-13129 (2005).

11 Zhang, G., Palmer, G. M., Dewhirst, M. W. \& Fraser, C. L. A dual-emissive-materials design concept enables tumour hypoxia imaging. Nat. Mater. 8, 747-751 (2009).

12 Palmer, G. M., Fontanella, A. N., Zhang, G., Hanna, G., Fraser, C. L. \& Dewhirst, M. W. Optical imaging of tumor hypoxia dynamics. J. Biomed. Opt. 15, 066021 (2010).

13 Samonina-Kosicka, J., DeRosa, C. A., Morris, W. A., Fan, Z. \& Fraser, C. L. Dualemissive difluoroboron naphthyl-phenyl $\beta$-diketonate polylactide materials: effects of heavy atom placement and polymer molecular weight. Macromolecules 47 3736-3746 (2014).

14 DeRosa, C. A., Samonina-Kosicka, J., Fan, Z., Hendargo, H. C., Weitzel, D. H., Palmer, G. M. \& Fraser, C. L. Oxygen sensing difluoroboron dinaphthoylmethane polylactide. Macromolecules 48, 2967-2977 (2015).

15 Hirose, A., Tanaka, K., Tamashima, K. \& Chujo, Y. Synthesis for dual-emissive organometallic complexes containing heterogeneous metal elements. Tetrahedron Lett. 55, 6477-6481 (2014).

16 Turanova, O. A., Garifzyanova, G. G. \& Turanov, A. N. Liquid crystal polymorphism of boron difluoride $\beta$-diketonates. Russ. J. Gen. Chem. 80, 2317-2322 (2010).

17 Turanova, O. A., Kal'dyaeva, E. V., Gnezdilov, O. I., Nikitin, S. I. \& Turanov, A. N. The first mesogenic derivative of boron difluoride $\pi$-diketonate. Russ. J. Gen. Chem. 76 730-732 (2006)

18 Sánchez, I., Mayoral, M. J., Ovejero, P., Campo, J. A., Heras, J. V., Cano, M. \& Lodeiro, C. Luminescent liquid crystal materials based on unsymmetrical boron difluoride $\beta$-diketonate adducts. New J. Chem. 34, 2937-2942 (2010).

19 Sánchez, I., Núñez, C., Campo, J. A., Torres, M. R., Cano, M. \& Lodeiro, C. Polycatenar unsymmetrical $\beta$-diketonate ligands as a useful tool to induce columnar mesomorphism on highly luminescent boron difluoride complexes. J. Mater. Chem. C 2 9653-9665 (2014).
20 Maeda, H., Naritani, K., Honsho, Y. \& Seki, S. Anion modules: Building blocks of supramolecular assemblies by combination with $\pi$-conjugated anion receptors. J. Am Chem. Soc. 133, 8896-8899 (2011).

21 Bando, Y., Sakurai, T., Seki, S. \& Maeda, H. Corannulene-fused anion-responsive $\pi$-conjugated molecules that form self-assemblies with unique electronic properties. Chem. Asian J. 8, 2088-2095 (2013).

22 Maeda, H., Terashima, Y., Haketa, Y., Asano, A., Honsho, Y., Seki, S., Shimizu, M., Mukai, H. \& Ohta, K. Discotic columnar mesophases derived from 'rod-like' $\pi$-conjugated anion-responsive acyclic oligopyrroles. Chem. Commun. 46 4559-4561 (2010)

23 Haketa, Y. \& Maeda, H. From helix to macrocycle: anion-driven conformation control of $\pi$-conjugated acyclic oligopyrroles. Chem. Eur. J. 17, 1485-1492 (2011).

24 Guieu, S., Pinto, J., Silva, V. L. M., Rocha, J. \& Silva, A. M. S. Synthesis, postmodification and fluorescence properties of boron diketonate complexes. Eur. J. Org. Chem. 2015, 3423-3426 (2015)

25 Xu, S., Evans, R. E., Liu, T., Zhang, G., Demas, J. N., Trindle, C. O. \& Fraser, C. L. Aromatic difluoroboron $\beta$-diketonate complexes: effects of $\pi$-conjugation and media on optical properties. Inorg. Chem. 52, 3597-3610 (2013).

26 Ono, K., Yoshikawa, K., Tsuji, Y., Yamaguchi, H., Uozumi, R., Tomura, M., Taga, K. \& Saito, K. Synthesis and photoluminescence properties of $\mathrm{BF}_{2}$ complexes with 1,3diketone ligands. Tetrahedron 63, 9354-9358 (2007).

27 Cogné-Laage, E., Allemand, J.-F., Ruel, O., Baudin, J.-B., Croquette, V., Blanchard-Desce, M. \& Jullien, L. Diaroyl(methanato)boron difluoride compounds as medium-sensitive two-photon fluorescent probes. Chem. Eur. J. 10, 1445-1455 (2004).

28 Mirochnik, A. G., Fedorenko, E. V. \& Karasev, V. E. Size-dependent luminescence of boron difluoride $\beta$-diketonates. Russ. Chem. Bull. Int. Ed. 57, 1190-1193 (2008).

29 Wang, D.-J., Pi, Y., Xu, B.-P., Wei, X.-H. \& Hu, Y.-J. A new difluoroboron bis- $\beta-$ diketonate with high quantum yield and intense photoluminescence. Optoelectron. Adv. Mat. 9, 79-82 (2015).

$30 \mathrm{Pi}$, Y., Wang, D.-J., Liu, H., Hu, Y.-J., Wei, X.-H. \& Zheng, J. Synthesis and spectroscopic properties of some new difluoroboron bis- $\beta$-diketonate derivatives. Spectrochim. Acta A 131, 209-213 (2014).

31 Wang, D.-J., Kang, Y.-F., Xu, B.-P., Zheng, J. \& Wei, X.-H. Synthesis, characterization and fluorescence properties of boron difluoride pyridyl- $\beta$-diketonate derivatives. Spectrochim. Acta A 104, 419-422 (2013)

32 Fedorenko, E. V., Mirochnik, A. G., Beloliptsev, A. Y. \& Isakov, V. V. $\left(\mathrm{S}_{2} \rightarrow \mathrm{S}_{0}\right)$ and $\left(\mathrm{S}_{1} \rightarrow \mathrm{S}_{0}\right)$ luminescence of dimethylaminostyryl- $\beta$-diketonates of boron difluoride. Dyes Pigments 109, 181-188 (2014).

33 Vovna, V. I., Tikhonov, S. A. \& Lvov, I. B. Photoelectron spectra and electronic structure of boron difluoride $\beta$-diketonates with aromatic substituents. Russ. J. Phys. Chem. A 87, 688-693 (2013).

34 Ono, K., Nakashima, A., Tsuji, Y., Kinoshita, T., Tomura, M., Nishida, J. \& Yamashita, Y. Synthesis and properties of terthiophene and bithiophene derivatives functionalized by $\mathrm{BF}_{2}$ chelation: a new type of electron acceptor based on quadrupolar structures. Chem. Eur. J. 16, 13539-13546 (2010).

35 Poon, C.-T., Lam, W. H. \& Yam, W.-W. Synthesis, photochromic, and computational studies of dithienylethene-containing $\beta$-diketonate derivatives and their near-infrared photochromic behavior upon coordination of a boron(III) center. Chem. Eur. J. 19, 3467-3476 (2013)

36 Poon, C.-T., Lam, W. H., Wong, H.-L. \& Yam, W.-W. A versatile photochromic dithienylethene-containing $\beta$-diketonate ligand: near-infrared photochromic behavior and photoswitchable luminescence properties upon incorporation of a boron(III) center. J. Am. Chem. Soc. 132, 13992-13993 (2010).

37 Maeda, H., Bando, Y., Shimomura, K., Yamada, I., Naito, M., Nobusawa, K., Tsumatori, H. \& Kawai, T. Chemical-stimuli-controllable circularly polarized luminescence from anion-responsive $\pi$-conjugated molecules. J. Am. Chem. Soc. 133 9266-9269 (2011)

38 Kumar, G. R. \& Thilagar, P. Triarylborane conjugated acacH ligands and their BF complexes: facile synthesis and intriguing optical properties. Dalton Trans. 43, 3871-3879 (2014).

39 Koch, M., Perumal, K., Blacque, O., Garg, J. A., Saiganesh, R. Kabilan, S. Balasubramanian, K. K. \& Venkatesan, K. Metal-free triplet phosphors with high emission efficiency and high tunability. Angew. Chem. Int. Ed. 53, 6378-6382 (2014).

40 Perumal, K., Garg, J. A., Blacque, O., Saiganesh, R., Kabilan, S., Balasubramanian, K. K. \& Venkatesan, K. $\beta$-Iminoenamine- $B F_{2}$ complexes: aggregation-induced emission and pronounced effects of aliphatic rings on radiationless deactivation. Chem. Asian J. 7, 2670-2677 (2012)

41 Zhang, Z., Wu, Z., Sun, J., Yao, B., Zhang, G., Xue, P. \& Lu, R. Mechanofluorochromic properties of $\beta$-iminoenolate boron complexes tuned by the electronic effects of terminal phenothiazine and phenothiazine-S,S-dioxide. J. Mater. Chem. C 3, 4921-4932 (2015).

42 Zhang, Z., Xue, P., Gong, P., Zhang, G., Peng, J. \& Lu, R. Mechanofluorochromic behaviors of $\beta$-iminoenolate boron complexes functionalized with carbazole. J. Mater. Chem. C 2, 9543-9551 (2014).

43 Ito, F. \& Sagawa, T. Quantitative evaluation of thermodynamic parameters for thermal back-reaction after mechanically induced fluorescence change. RSC Adv. 3 , 19785-19788 (2013)

44 Zhang, G., Lu, J., Sabat, M. \& Fraser, C. L. Polymorphism and reversible mechanochromic luminescence for solid-state difluoroboron avobenzone. J. Am. Chem. Soc. 132, 2160-2162 (2010).

45 Wang, L., Wang, K., Zou, B., Ye, K., Zhang, H. \& Wang, Y. Luminescent chromism of boron diketonate crystals: distinct responses to different stresses. Adv. Mater. 27 2918-2922 (2015) 
46 Luo, J. D., Xie, Z. L., Lam, J. W. Y., Cheng, L., Chen, H. Y., Qiu, C. F., Kwok, H. S., Zhan, X. W., Liu, Y. Q., Zhu, D. B. \& Tang, B. Z. Aggregation-induced emission of 1-methyl-1,2,3,4,5-pentaphenylsilole. Chem. Commun. 1740-1741 (2001).

47 Yoshii, R., Nagai, A., Tanaka, K. \& Chujo, Y. Highly emissive boron ketoiminate derivatives as new class of aggregation-induced emission fluorophores. Chem. Eur. J. 19, 4506-4512 (2013).

48 Macedo, F. P., Gwengo, C., Lindeman, S. V., Smith, M. D. \& Gardinier, J. R. $\beta$-Diketonate, $\beta$-ketoiminate, and $\beta$-diiminate complexes of difluoroboron. Eur. J. Inorg. Chem. 2008, 3200-3211 (2008).

49 Yoshii, R., Suenaga, K., Tanaka, K. \& Chujo, Y. Mechanofluorochromic materials based on aggregation-induced emission-active boron ketoiminates: regulation of the direction of the emission color changes. Chem. Eur. J. 21, 7231-7237 (2015).

50 Kubota, Y., Tanaka, S., Funabiki, K. \& Matsui, M. Synthesis and fluorescence properties of thiazole-boron complexes bearing a $\beta$-ketoiminate ligand. Org. Lett. 14, 4682-4685 (2012).

51 Liao, C.-W., Rao, M. R. \& Sun, S.-S. Structural diversity of new solid-state luminophores based on quinoxaline- $\beta$-ketoiminate boron difluoride complexes with remarkable fluorescence switching properties. Chem. Commun. 51, 2656-2659 (2015).

52 Wu, Y., Li, Z., Liu, Q., Wang, X., Yan, H., Gong, S., Liu, Z. \& He, W. High solid-state luminescence in propeller-shaped AIE-active pyridine-ketoiminate-boron complexes. Org. Biomol. Chem. 13, 5775-5782 (2015).

53 Galer, P., Korošec, R. C., Vidmar, M. \& Šket, B. Crystal structures and emission properties of the $\mathrm{BF}_{2}$ complex 1-phenyl-3-(3,5-dimethoxyphenyl)-propane-1,3-dione: multiple chromisms, aggregation- or crystallization-induced emission, and the selfassembly effect. J. Am. Chem. Soc. 136, 7383-7394 (2014).

54 Yoshii, R., Hirose, A., Tanaka, K. \& Chujo, Y. Boron diiminate with aggregation-induced emission and crystallization-induced emission enhancement characteristics. Chem. Eur. J. 20, 8320-8324 (2014).

$55 \mathrm{Bu}$, J., Watanabe, K., Hayasaka, H. \& Akagi, K. Photochemically colour-tuneable white fluorescence illuminants consisting of conjugated polymer nanospheres. Nat. Commun. 5, 3799 (2014)

56 Tanaka, K. \& Chujo, Y. Advanced luminescent materials based on organoboron polymers. Macromol. Rapid Commun. 33, 1235-1255 (2012).

57 Nagai, A., Kokado, K., Nagata, Y. \& Chujo, Y. 1,3-Diketone-based organoboron polymers: Emission by extending conjugation along a polymeric ligand. Macromolecules 41, 8295-8298 (2008).
58 Nagai, A., Kokado, K., Nagata, Y., Arita, M. \& Chujo, Y. Highly intense fluorescent diarylboron diketonate. J. Org. Chem. 73, 8605-8607 (2008).

59 Tanaka, K., Tamashima, K., Nagai, A., Okawa, T. \& Chujo, Y. Facile modulation of optical properties of diketonate-containing polymers by regulating complexation ratios with boron. Macromolecules 46, 2969-2975 (2013).

60 Yoshii, R., Nagai, A., Tanaka, K. \& Chujo, Y. Boron ketoiminate-based polymers: finetuning of the emission color and expression of srong emission both in the solution and film state. Macromol. Rapid Commun. 35, 1315-1319 (2014).

61 Yoshii, R., Tanaka, K. \& Chujo, Y. Conjugated polymers based on tautomeric units: regulation of main-chain conjugation and expression of aggregation induced emission property via boron-complexation. Macromolecules 47, 2268-2278 (2014).

62 Jiang, X., Liu, X., Jiang, Y., Quan, Y., Cheng, Y. \& Zhu, C. Fluorescence study of chiral $\beta$-detoiminate-based newly synthesized boron hybrid polymers. Macromol. Rapid Commun. 215, 358-364 (2014).

63 Dai, C., Yang, D., Fu, X., Chen, Q., Zhu, C., Cheng, Y. \& Wang, L. Study on tunable AIE (AIEE) of boron ketoiminate-based conjugated polymers for live cell imaging. Polym. Chem. 6, 5070-5076 (2015).

64 Yoshii, R., Hirose, A., Tanaka, K. \& Chujo, Y. Functionalization of boron diiminates with unique optical properties: multicolor tuning of crystallization-induced emission and introduction into the main-chain of conjugated polymers. J. Am. Chem. Soc. 136, 18131-18139 (2014).

65 Hirose, A., Tanaka, K., Yoshii, R. \& Chujo, Y. Film-type chemosensors based on boron diminate polymers having oxidation-induced emission properties. Polym. Chem. 6, 5590-5595 (2015).

This work is licensed under a Creative Commons Attribution 4.0 International License. The images or other third party material in this article are included in the article's Creative Commons license, unless indicated otherwise in the credit line; if the material is not included under the Creative Commons license, users will need to obtain permission from the license holder to reproduce the material. To view a copy of this license, visit http:// creativecommons.org/licenses/by/4.0/ 